\title{
A NOVEL PHASE TRANSITION IN ALLOYS
}

$\mathrm{ANL} / \mathrm{CP}--74212$

DE92 000366

\author{
Marie Louise Saboungi and David L. Price \\ Materials Science Division \\ Argorne National Laboratory \\ Argonne, IL 60439
}

\begin{abstract}
The submined manuscript has been authored by a
contractor of the U.S. Government under contract No.

W.31-109-ENG-38. Accordingly, the U.S. Government

retains a nonexclusive, royalty-iree license to publish

o reproduce the published lorm of this contribution, or

allow others 10 do so. for U.S. Government purposes.
\end{abstract}

To be published in J. Non-Cryst. Solids

September, 1991

$/ \mathrm{sm}$

Distribution:
1-3. D. Hamrin
4. B. D. Dunlap
5. M. B. Brodsky
6. J. D. Jorgensen
7. B. S. Brown
8. Editorial Office
9. Authors

\section{DISCLAIMER}

\begin{abstract}
This report was prepared as an account of work sponsored by an agency of the United States Government. Neither the United States Government nor any agency thereof, nor any of their employees, makes any warranty, express or implied, or assumes any legal liability or responsibility for the accuracy, completeness, or usefulness of any information, apparatus, product, or process disclosed, or represents that its use would not infringe privately owned rights. Reference herein to any specific commercial product, process, or service by trade name, trademark, manufacturer, or otherwise does not necessarily constitute or imply its endorsement, recommendation, or favoring by the United States Government or any agency thereof. The views and opinions of authors expressed herein do not necessarily state or reflect those of the United States Government or any agency thereof.
\end{abstract}

Invited Paper for the 5th International Conference on Structure of Non-Crystalline Materials, Sendai, Japan, Sept. 2-6, 1991

This work is supported by the U.S. Department of Energy, Basic Energy Sciences, Materials Sciences, under contract No. W-31-109-ENG-38. 


\title{
A NOVEL PHASE TRANSITION IN ALlOYS
}

\author{
Marie Louise Saboungi and David L. Price \\ Materials Science Division \\ Argonne National Laboratory \\ Argonne, IL 60439
}

\begin{abstract}
The submited manuscript has been authored by a
contractor of the U.S. Government under contract No.

W-31-109-ENG-38. Accordingly, the U.S. Government

retains a nonexclusive, foyalty-free license to publish

of reproduce the publisted form of this contrbution, or

aflow others to do so. for U.S. Covernment purposes.
\end{abstract}

To be published in J. Non-Cryst. Solids

September, 1991

$/ \mathrm{sm}$

Invited Paper for the 5th Iniernational Conference on Structure of Non-Crystalline Materials, Sendai, Japan, Sept. 2-6, 1991

This work is supported by the U.S. Department of Energy, Basic Energy Sciences, Materials Sciences, under contract No. W-31-109-ENG-38. 


\title{
A NOVEL PHASE TRANSITION IN ALLOYS
}

\author{
Marie-Louise Saboungi and David Long Price \\ Materials Science Division \\ Argonne National Laboratory \\ Argonne, Il 60439 \\ Invited Paper for 5th International Conference on \\ Structure of Non-Crystalline Materials \\ Sendai, Japan \\ September 2-6, 1991
}

To be published in J. Non-Cryst. Solids 
A NOVEL PHASE TRANSITION IN ALLOYS

MARIE-LOUISE SABOUNGI and DAVID LONG PRICE

Materials Science Division, Argonne National Laboratory, Argonne, IL 60439 USA

The intermediate-phase of the intermetallic alloy $\mathrm{CsPb}$ is shown to be a plastic crystal characterized by jump reorientations of $\mathrm{Cs}_{4} \mathrm{~Pb}_{4}$ structural units. The wave vector variation of elastic and quasielastic intensities is well reproduced by a simple model of independent structural units jumping between the four orientations observed in the crystal at room temperature. This represents the first observation of a plastic crystal phase in a metal alloy.

\section{INTRODUCTION}

Recent work on the structure of condensed matter has blurred the conventional distinction between crystalline and non-crystalline materials. On the one hand, materials traditionally considered to be disordered, such as glasses and liquids, shown an appreciable amount of regularity in their structure. Even liquid metals exhibit a wide variety of types of short- and intermediate-range order ${ }^{1}$. At the same time, correlations in atomic disorder are being increasingly recognized as playing an important role in crystalline materials, for example in ferroelectric and superconducting oxides. ${ }^{2}$ In categorizing the different types of order and disorder involved, it is often useful to distinguish between translational and orientational order. Figure 1 shows a schematic plot of different types of condensed matter on this basis.

In the case of metal alloys, the recently discovered quasicrystals can be treated as solids exhibiting orientational long-range order (LRO) but without translational LRO. The purpose of this paper is to show that the opposite situation my occur in a metal alloy, namely translational LRO without orientational LRO. Though conclusively established so far in only one alloy, $\mathrm{CsPb}$, other cases are very likely to be found.

\section{ALKALI-LEAD ALLOYS}

Equiatomic alloys of alkali metals with lead and tin form highly ordered structures in both the solid and liquid states ${ }^{3}$. Transfer of approximately one electron per atom from the alkali to the heavy metal atom leads to covalent bonding and the formation of tetrahedral $\mathrm{Pb}_{4}^{4-}$ or $\mathrm{Sn}_{4}^{4-}$

complexes. The existence of these complexes, first proposed by Zintl in the 1930's, explains the 
unusual structures of these alloys in solid and liquid states and their remarkable electrical transport ${ }^{4}$ and thermodynamic 5 behavior.

The systematics of the alkali metal-lead alloys $\mathrm{APb}$ have been studied with neutron diffraction ${ }^{3}$, electrical resistivity ${ }^{4}$ and calorimetric measurements ${ }^{6}$. The crystal structures in the solid can be described in terms of $\mathrm{Pb}_{4}$ tetrahedra surrounded by larger, oppositely directed $\mathrm{A}_{4}$ tetrahedra. The liquid structures are well represented in terms of the random packing of these $\mathrm{A}_{4} \mathrm{~Pb}_{4}$ units. As the size of the $\mathrm{A}$ atom increases, the structural units become more stable, and electron transfer take place more predominantly within the units rather than through the network as a whole ${ }^{1}$. These properties are most pronounced in the end member of the series, $\mathrm{CsPb}$, which has an unusually high resistivity $7,7,000 \mu \Omega \mathrm{cm}$, and large negative temperature coefficient, -115 $\mu \Omega \mathrm{cm} / \mathrm{K}$, in the liquid, characteristic of a type-III semiconductor in the scheme of Mott and Davis ${ }^{8}$.

$\mathrm{CsPb}$ is unusual also in its melting behavior, which appears to take place in two stages. The calorimetric results of Saboungi et al 6 , reproduced here in Figure 2, revealed two transitions at $869 \mathrm{~K}$ and $920 \mathrm{~K}$, with comparable entropy changes, $\Delta S=0.86 \mathrm{R}$ and $0.52 \mathrm{R}$, respectively. In contrast, $\mathrm{KPb}$ has only a single transition on melting. A behavior similar to that of $\mathrm{CsPb}$ was noticed in NaSn by Hume-Rothery and ascribed to a "polymorphic transition of the compound NaSn." 9 In the expectation that the lower transition in $\mathrm{CsPb}$ was of this type, neutron diffraction measurements were made at $880 \mathrm{~K}$ in the intermediate phase during the work described in Ref. 3 . The measured structure factor, shown in Figure 3, resembles to a large extent that taken in the liquid at $930 \mathrm{~K}$. However, the first diffraction peak is sharper and more pronounced than in the liquid and can be ascribed to a Bragg reflection (or two unresolved reflections) broadened by the resolution of the diffractometer. However, no further Bragg peaks can be identified, making it impossible to determine the crystal structure of this phase if, indeed, it exists.

The fact that this behavior is observed only by $\mathrm{CsPb}$ out of all the alkali metal-lead alloys indicates that a steric factor may be involved. The larger size of the $\mathrm{Cs}$ atom makes the $\mathrm{Cs}_{4} \mathrm{~Pb}_{4}$ structural units more spherical and more capable of rotation ${ }^{10}$, suggesting the possibility that the intermediate phase may be a plastic crystal with structural units located on a crystal lattice while undergoing rapid reorientation.

\section{QUASIELASTIC NEUTRON SCATTERING}

To investigate this possibility, quasielastic scattering measurements on $\mathrm{CsPb}$ were carried out on the IN6 spectrometer ${ }^{11}$ at the Institut Laue-Langevin (ILL). An incident neutron wavelength of 
$5.12 \AA$ was used, enabling quasielastic measurements out to a wavevector $Q=2.05 \AA^{-1}$, just below the maximum of the second peak in $S(Q)$. Measurements were made in the low-temperature phase at $300 \mathrm{~K}$ and $833 \mathrm{~K}$, in the intermediate phase at $873 \mathrm{~K}, 888 \mathrm{~K}$ and $903 \mathrm{~K}$, and in the liquid at $923 \mathrm{~K}$. At $873 \mathrm{~K}$ two runs were made, before and after melting the sample: no discernible difference was observed. Time-of-flight data were collected in 19 groups of detectors with scattering angles ranging from $16.6^{\circ}$ to $112.9^{\circ}$, equivalent to a $Q$ range for elastic scattering of $0.35-2.05 \AA^{-1}$.

Representative quasielastic energy spectra for the three phases are shown in Figure 4. It is seen that the material at $833 \mathrm{~K}$, below the first transition, behaves as a normal crystalline solid with no hint of line broadening. At $923 \mathrm{~K}$, above the upper transition, it behaves as a liquid, with no hint of elastic scattering, although at least two Lorentzian functions appear necessary to fit the detailed quasielastic spectra. The data at $903 \mathrm{~K}$, intermediate between the two transitions, are characterized by a superposition of elastic and broadened quasielastic scattering which can be satisfactorily fitted with a delta function and single Lorentzian function, each broadened with the instrumental resolution as given by a vanadium measurement.

Figure 5 shows the $Q$ variation of the intensities of the fitted functions for the four runs in the intermediate phase. It is seen that the quasielastic intensity has a small peak at $Q=0.90 \AA^{-1}$ : slightly below the $Q$ value of the first peak in $S(Q)$ at $0.97 \AA^{-1}$, and starts to rise again at $1.3 \AA^{-1}$, comprising most of the strength of the second peak in $S(Q)$. The elastic intensity accounts for most of the strength of the first peak in $S(Q)$ with small, but significant, values elsewhere. The general form of these data is strongly suggestive of molecular reorientations and supports the identification of the intermediate phase as a plastic crystal.

\section{JUMP REORIENTATION MODEL}

To confirm this interpretation and identify the nature of the reorienting units and the geometry of the reorientations, a detailed dynamical model is required. The structure factor for coherent scattering from an assembly of structural units is given by 12

$$
S(Q)=f_{1}(Q)+f_{2}(Q)\left[S_{c}(Q)-1\right]
$$

where $f_{1}(Q)$ is the form factor of the unit, $f_{2}(Q)$ another form factor expressing the average correlation between two distinct units, and $S_{c}(Q)$ the structure factor describing the arrangement of the centers of the units. For the present study, we assume that the reorientations of each unit are uncorrelated, so the quasielastic scattering is confined to the first term of Eq. (1). 
The room-temperature crystal structure of $\mathrm{CsPb}$ is analogous to that of $\mathrm{NaPb}{ }^{3,13}$ The unit cell contains four $\mathrm{Cs}_{4} \mathrm{~Pb}_{4}$ structural units oriented in four non-equivalent directions. If the units undergo stochastic jumps between these four orientations, the frequency dependence of the first term in Eq. (1) can be shown to be given by 14

$$
S_{1}(Q, \omega)=\sum_{j \mathbf{j}} a_{j k} \rho_{1}(\mathbf{Q})^{*} \rho_{j}(\mathbf{Q})\left(\frac{\omega_{k} / \pi}{\omega^{2}+\omega_{k}^{2}}\right)
$$

summed over the four orientations $j$, where

$$
\rho_{j}(\mathbf{Q})=\left\langle\frac{1}{\bar{b}^{2}}\right\rangle \sum_{n=1}^{N} \bar{b}_{n} \exp \left(i \mathbf{Q} \bullet \mathbf{r}_{n}\right)
$$

summed over the atoms $\mathrm{n}$ in the structural unit, and the $\mathrm{a}_{\mathrm{jk}}$ and $\omega_{\mathrm{k}}$ are obtained by solving four coupled rate equations. ${ }^{14}$ The term $k=1$ in Eq. (2) has $\omega_{1}=0$, corresponding to elastic scattering, while the three remaining terms have nori-zero $\omega_{\mathrm{k}}$, giving rise to quasielastic scattering.

Integration of Eq. (5) over $\omega$ gives $\int_{-\infty}^{\infty} S_{1}(Q, \omega) d \omega=f_{1}(Q)$, the first term of Eq. (1), as required. The second term in Eq. (2) gives rise only to elastic scattering in this model.

Fig. 6 shows the integrated intensities for quasielastic and elastic scattering, calculated from this Eqs. (1) and (2), compared with the experimental data. The elastic scattering is broadened with a resolution function of width $\Delta \mathrm{Q}=0.1 \mathrm{Q}$. The model reproduces all the qualitative features of the data, including the first peak in the quasielastic intensity at $Q=0.9 \AA^{-1}$. The agreement can be considered satisfactory, especially since there are no adjustable parameters appearing in the calculated intensity. The first peak in the quasielastic intensity is a little lower than the measured one, indicating that orientational correlations between distinct units are making a small but significant contribution to the quasielastic scattering, neglected in the simple model discussed here. The energy widths of the quasielastic peaks are approximately $0.35 \mathrm{meV}$, consistent with the present model for jump times $\sim 8$ psec.

Calculations have also been made for different structural units, e.g. $\mathrm{Pb}_{4}^{4-}$, and different types of reorientation. For coherent quasielastic scattering, the results are strongly dependent on the 
choice of structural unit and reorientation geometry, unlike the more familiar case of incoherent quasielastic scattering. In particular, omission of the $\mathrm{Cs}$ atoms from the structural unit makes it impossible to reproduce the first peak in the quasielastic intensity at $Q=0.9 \AA^{-1}$. Also rotational diffusion, as opposed to discrete jump reorientations, does not lead to quasielastic scattering in the coherent case and can be ruled out of the dynamical picture.

Finally, we can now calculate $S(Q)$ for the intermediate phase from Eq. (1) and compare with the diffraction data of Figure 3. For the comparison, we have again broadened the calculated data with a Gaussian of width $\Delta \mathrm{Q}=0.1 \mathrm{Q}$ to obtain the solid curve shown in Figure 3. The agreement is seen to be excellent. This first peak is now seen to be a broadened Bragg peak, arising from two close reflections with plane spacing $\mathrm{d} \sim 6 \AA$. The remaining Bragg reflections are attenuated by the form factor $f_{2}(Q)$, and by the increased symmetry compared with the room-temperature phase, arising from the randomization of the orientations on a time-average basis.

\section{CONCLUSIONS}

It is clear from these data and the comparison with the model calculations that melting in $\mathrm{CsPb}$ takes place in two stages, the first to a state in which the structural units are translationally ordered but undergoing rapid jump reorientations, and the second to a true liquid in which the units are positionally disordered as well. The intermediate phase is plastic crystal which is a familiar state in organic molecular solids and in inorganic materials with stable complex ions ${ }^{15}$ but not, to our knowledge, encountered before in a metal alloy. The value of the entropy change at the transition, $\Delta S=0.86 \mathrm{R}$ per formula unit, is close to $\Delta \mathrm{S}=\mathrm{R} \ln 32 \mathrm{per} \mathrm{Cs}_{4} \mathrm{~Pb}_{4}$ structural unit, which is towards the high end of entropy gains observed in disordering transitions in salts. ${ }^{16}$ This may be understood on the basis of the model described in the last section if the $\mathrm{Pb}$ and $\mathrm{Cs}$ atoms reorient independently. In this case, each $\mathrm{Pb}_{4}$ tetrahedron and each nearest-neighbor $\mathrm{Cs}_{4}$ tetrahedron has four possible orientations, while a second-neighbor set of four $\mathrm{Cs}$ atoms arranged on a square concentric with the tetrahedra has two possible orientations, leading to a total of 32 distinguishable configurations. The total entropy change in the two transitions, $\Delta S=1.38 \mathrm{R}$ per formula unit, is close to $\Delta S=R \ln 2$ per atom which is a typical value of the entropy of melting for a broad range of materials. ${ }^{17}$

The results reported here indicate that the $\mathrm{Cs}$ atoms are participating in the reorientations. This is consistent with the structural data in both low-temperature solid and liquid. In the liquid, as we have shown above, inclusion of the $\mathrm{Cs}$ atoms in the structural unit in a model based on the random packing of structural units gives better agreement with the diffraction data. ${ }^{3}$ 
Finally, we make the experimental observation that coherent quasielastic scattering provides a powerful experimental technique for studying the orientations of molecules or structural units, being considerably more sensitive than the more familiar case of incoherent scattering for identifying the type of structural unit and reorientations involved.

\section{ACKNOWLEDGEMENTS}

The authors are grateful to Mr. R. Kleb for the elegant design of the sample container, Drs. D. Kearley and R. White and the staff of the Institut Laue-Langevin for making the experiments possible, and Prof. M. P. Tosi for helpful discussions. This work was performed under the auspices of the U.S. Department of Energy, Division of Materials Sciences, Office of Basic Energy Sciences, under Contract W-31-109-ENG-38.

\section{REFERENCES}

1. M.-L. Saboungi, W. Geertsma and D. L. Price, Annu. Rev. Phys. Chem. 41, (1990) 207.

2. T. Egami, H. D. Rosenfeld, B. H. Toby and A. Bhalla, Ferroelectrics, in press.

3. H. T. J. Reijers, M.-L. Saboungi, D. L. Price, J. W. Richardson, K. J. Volin and W. van der Lugt, Phys. Rev. B40, (1989) 6018; H. T. J. Reijers, M.-L. Saboungi, D. L. Price and W. van der Lugt, Phys. Rev. B41, (1990) 5661.

4. J. A. Meijer, W. Geertsma and W. van der Lugt, J. Phys. F: Metal Phys. 15 (1985) 899.

5. P. J. Tumidajski, A. Petric, T. Takenaka, A. D. Pelton and M.-L. Saboungi, J. Phys. Condens. Matter 2, (1990) 209.

6. G. K. Johnson and M.-L. Saboungi, J. Chem. Phys. 861987 6376; M.-L. Saboungi, H. T. J. Reijers, M. Blander and G. K. Johnson, J. Chem Phys. 89, (1988) 5869.

7. W. van der Lugt and J. A. Meijer, in: Amorphous and Liquid Materials, E. Lüscher, G. Fritzch and G. Jaccuci, Eds. (Martinus Nijhoff, Dordrecht, 1987) .

8. N. F. Mott and E. A. Davis, Electronic Processes in Non-Crystalline Materials (Clarendon Press, Oxford, 1979).

9. W. Hume-Rothery, J. Chem. Soc. 131, (1928) 947.

10. J. Woods Halley, private communication (1988).

11. R. Scherm, C. Carlile, A. J. Dianoux, J. B. Suck and J. W. White, ILL Report 76 S235 (1976).

12. P. A. Egelstaff, D. I. Page and J. G. Powles, Mol. Phys. 20, (1971) 881; 22, (1971) 994.

13. R. E. Marsh and D. P. Shoemaker, Acta Cryst. 6, (1953) 197.

14. D. L. Price and M.-L. Saboungi, Phys. Rev. B, in press. 
15. J. N. Sherwood, Ed., The Plastic Crystalline State (J. Wiley, Chichester, 1979); N. G. Parsonage and L. A. K. Staveley, Disorder in Crystals (Clarendon Press, Oxford, 1978).

15. D. M. Newns and L. A. K. Staveley, Chem. Rev. (London), 66 (1966) 267.

17. J. L. Tallon, Physics Letters 87A (1982) 361; ibid 365. 


\section{FIGURE CAPTIONS}

FIGURE 1. Types of order and disorder in condensed matter.

FIGURE 2. Enthalpy of $\mathrm{CsPb}$ and $\mathrm{KPb}$ as a function of temperature (Ref. 6).

FIGURE 3. Measured structure factor of $\mathrm{CsPb}$ at $880 \mathrm{~K}$ (circles). The solid line shows the structure factor for the model described in the text.

FIGURE 4. Quasielastic neutron spectra of CsPb for $41.7^{\circ}$ scattering angle at (a) $833 \mathrm{~K}$, (b) 903 $\mathrm{K}$ and (c) $923 \mathrm{~K}$ : data (circles), fitted delta functions (dotted lines), Lorentzians (dashed lines) and total scattering (solid lines).

FIGUVRE 5 Integrated intensity of delta functions and Lorentzian functions, fitted to quasielastic spectra, and total intensities as a function of wave vector $Q$. The points represent data from four separate runs and the lines connect the averaged values for the delta functions (dashed), Lorentzian functions (dotted) and total scattering (solid).

FIGURE 6. Measured intensities (circles) of (a) Lorentzian and (b) delta-function peaks, compared with intensities calculated from the model (solid lines). 


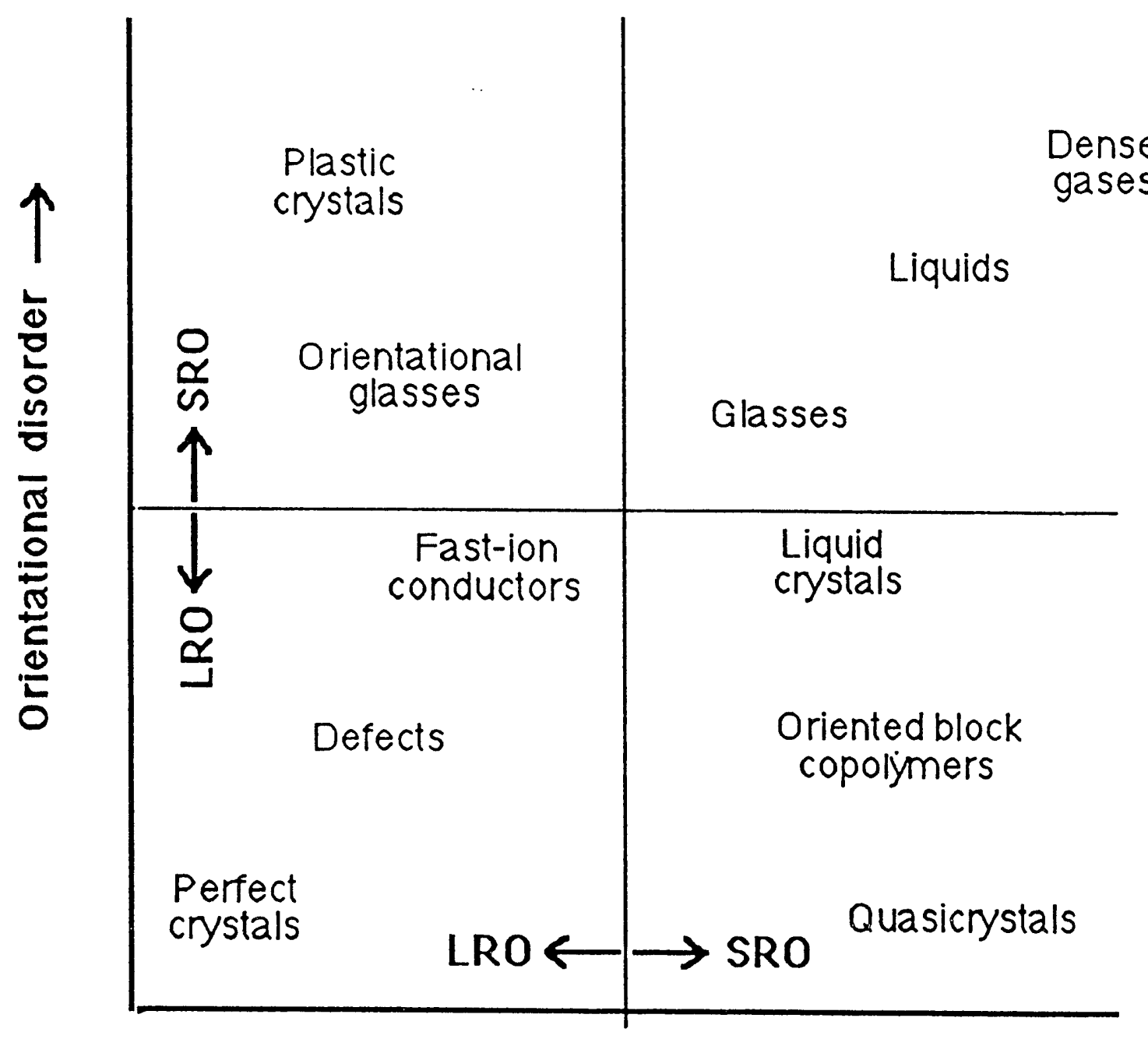

\section{Translational disorder $\longrightarrow$}




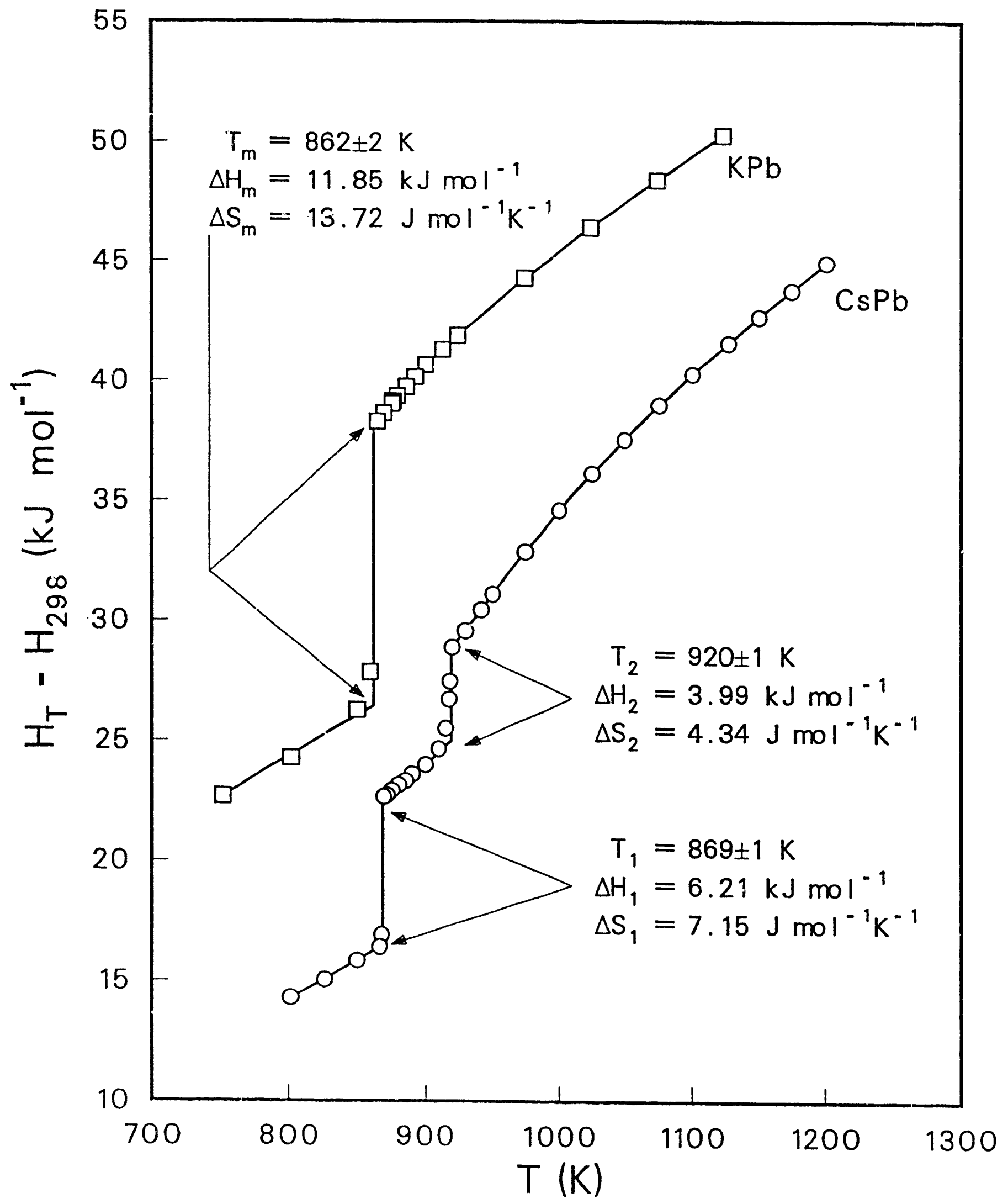




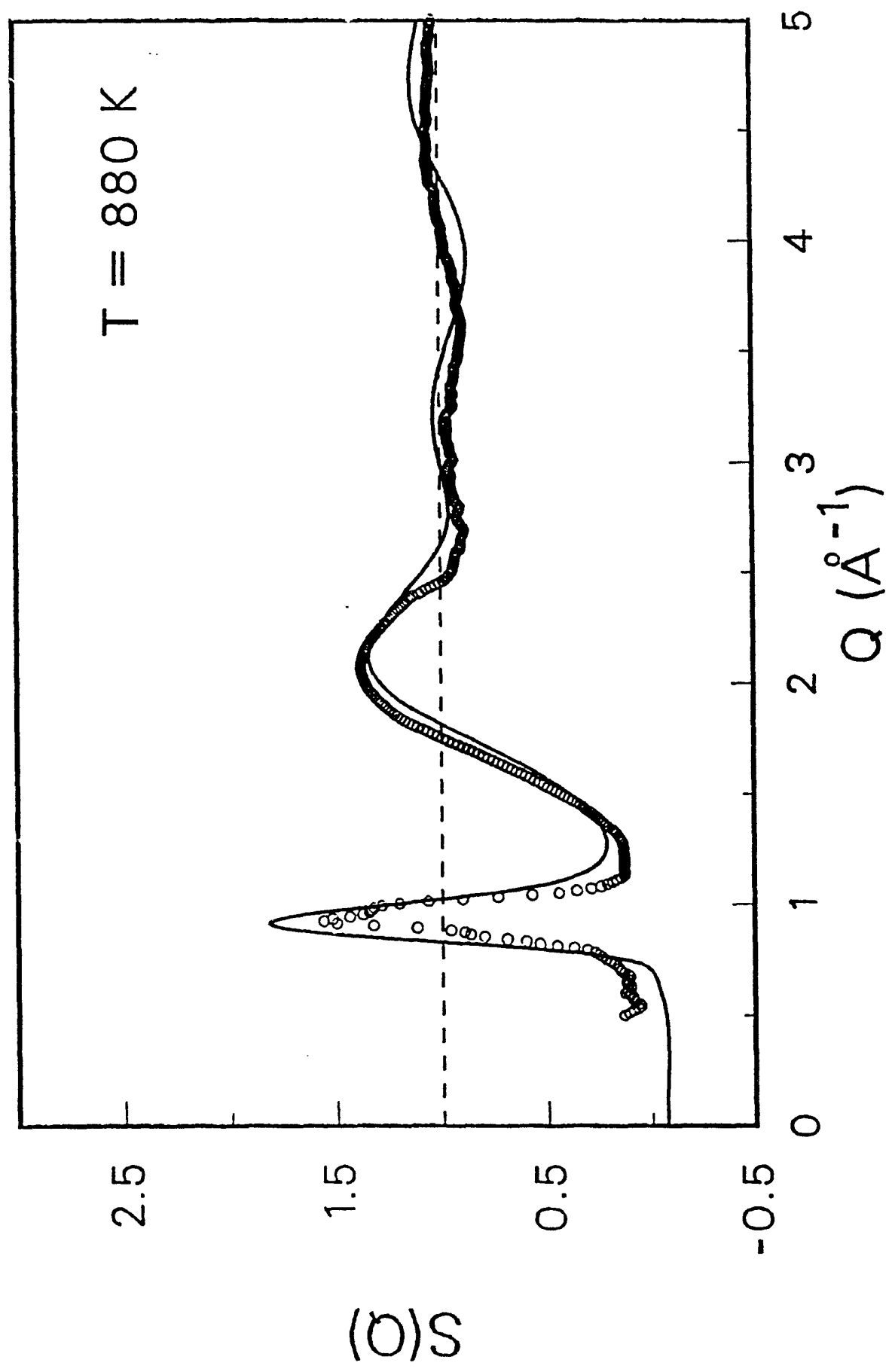



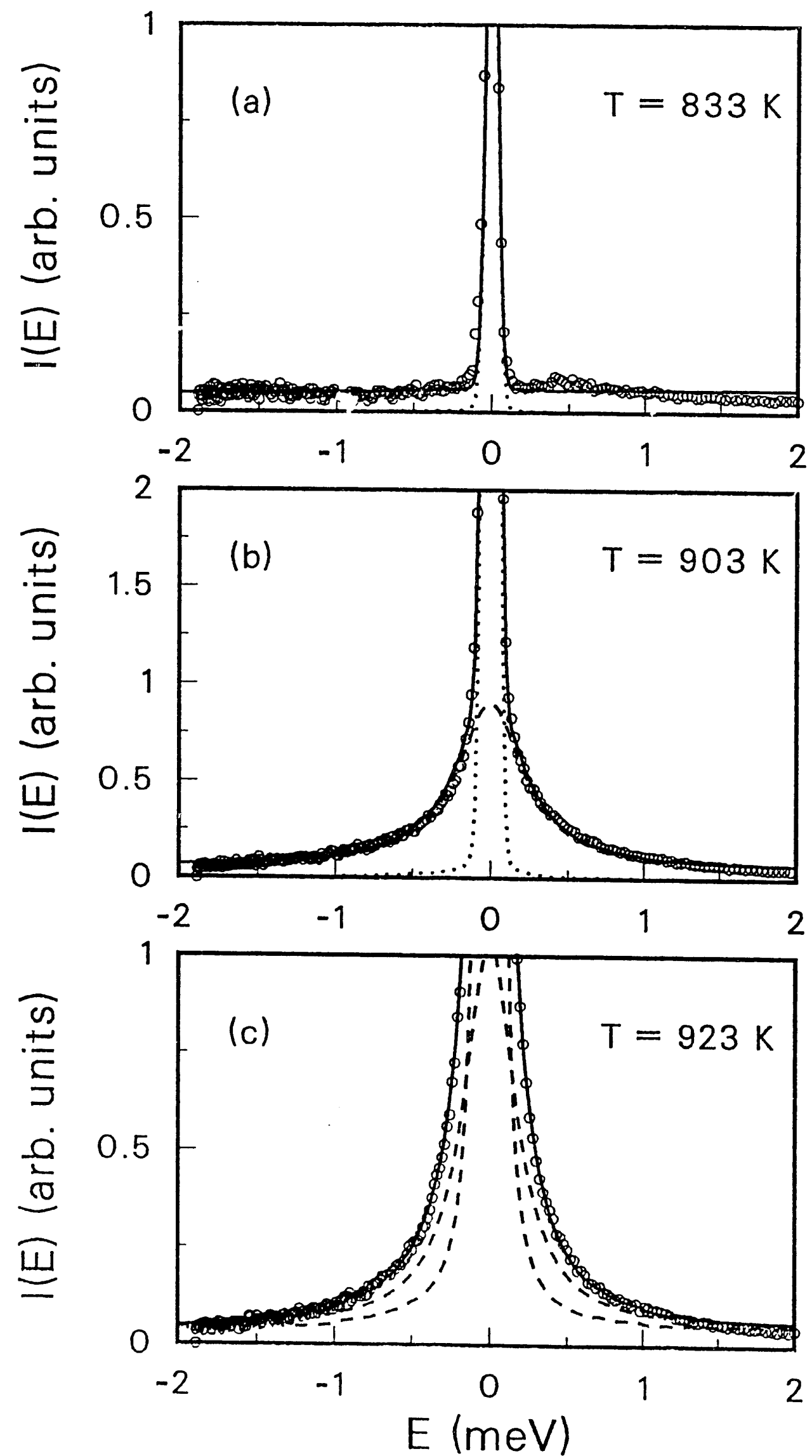


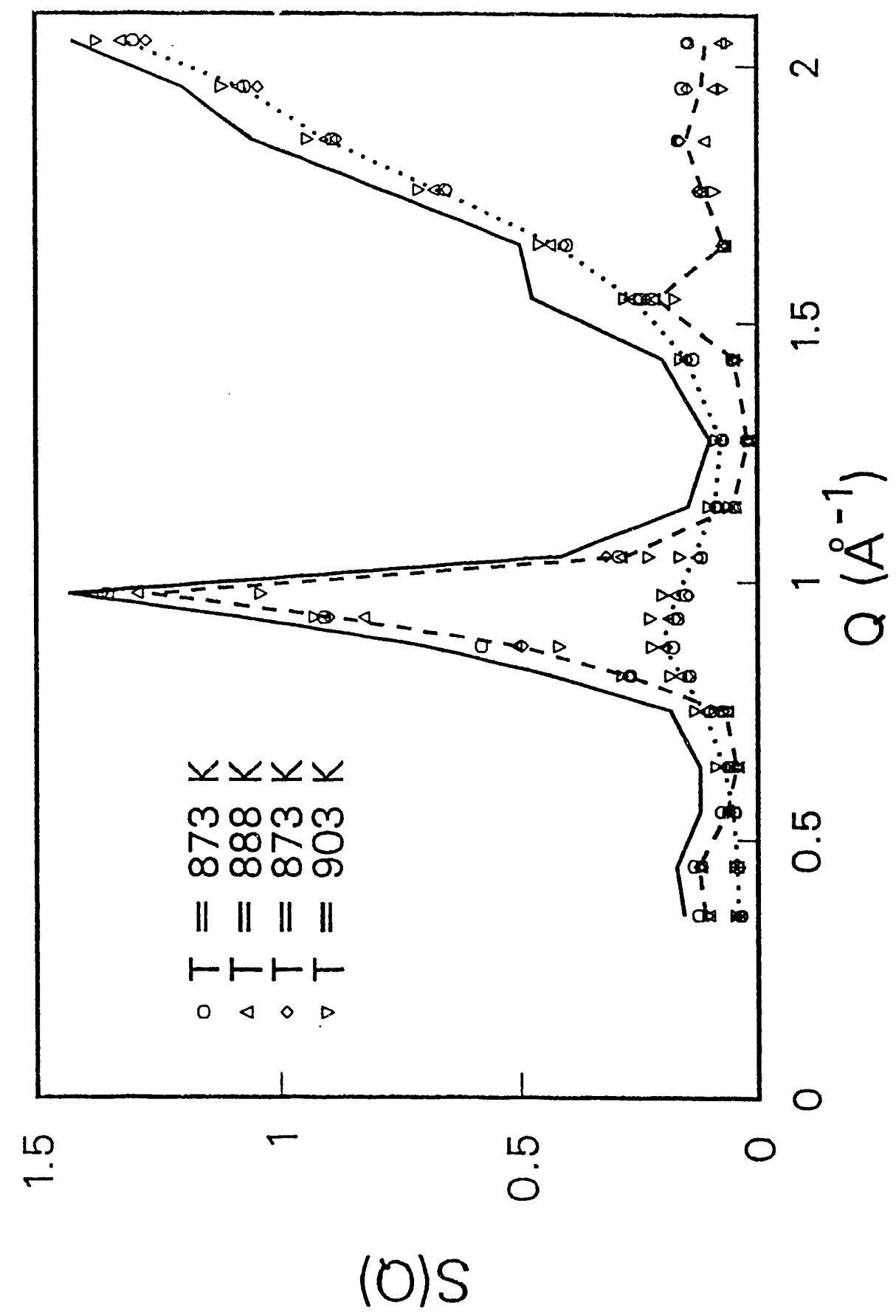



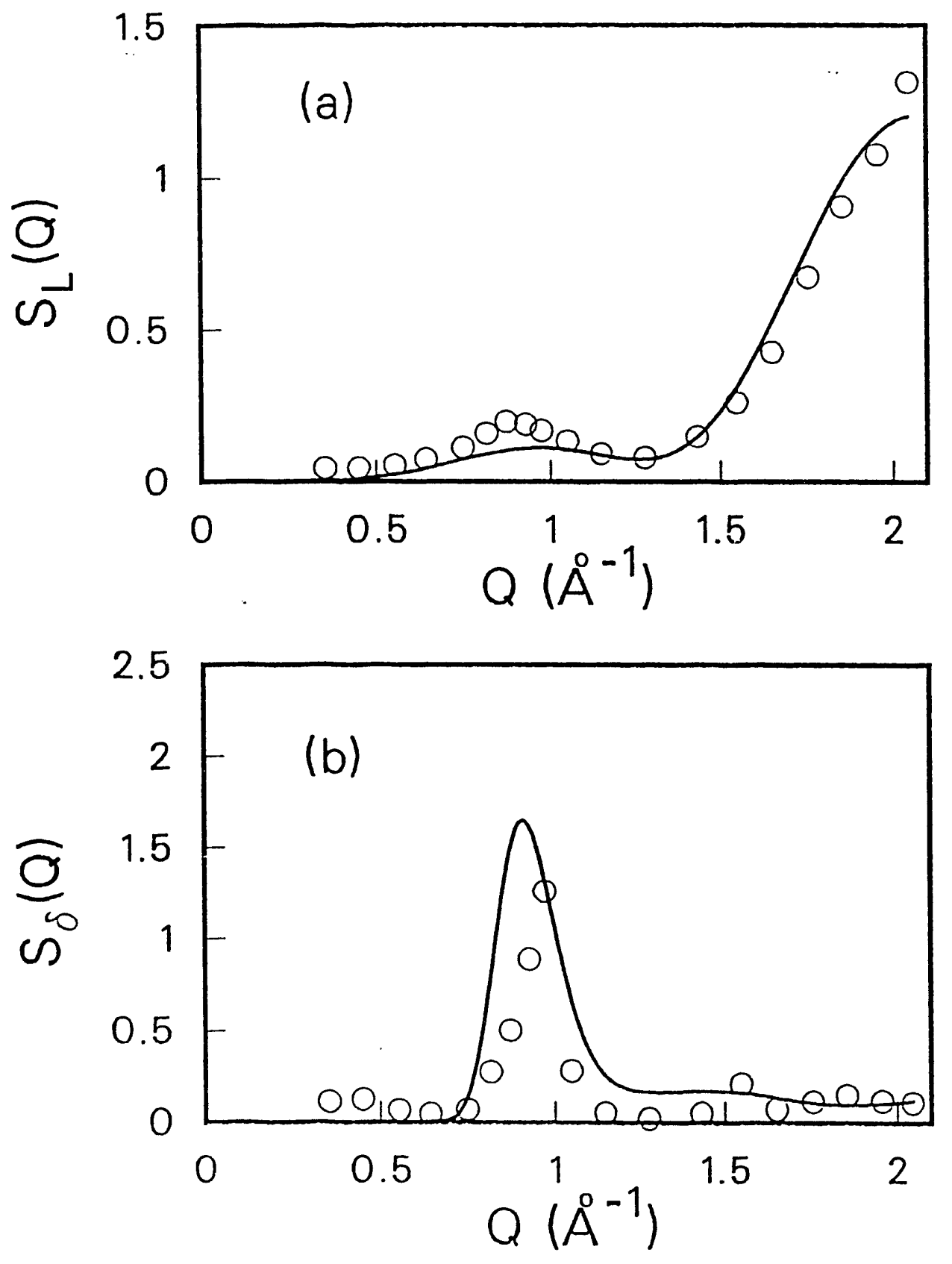

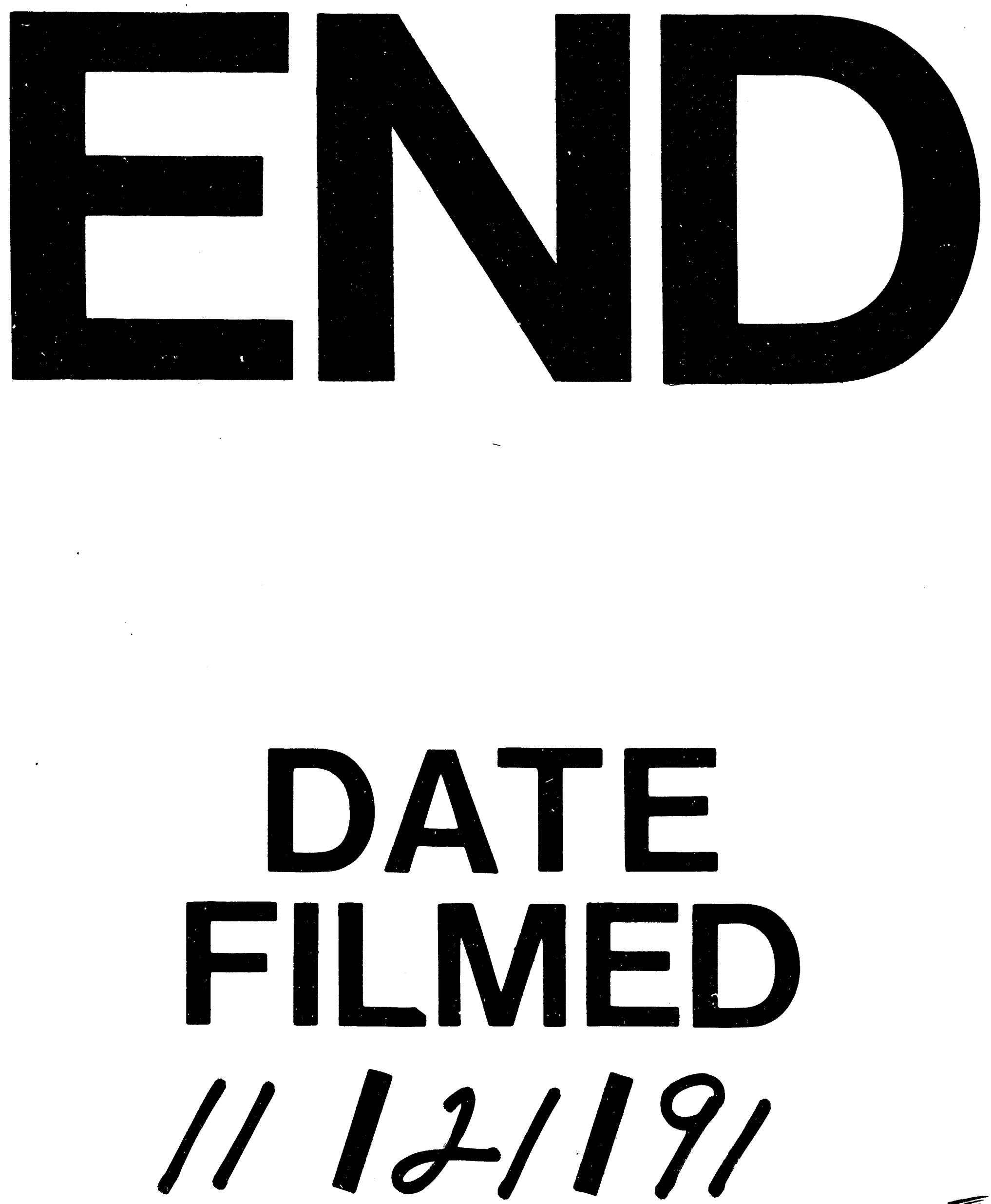

T" 
